\title{
Neuronal plasticity of the "brain-skin connection": stress-triggered up-regulation of neuropeptides in dorsal root ganglia and skin via nerve growth factor-dependent pathways
}

\author{
Ricarda A. Joachim • Arne Kuhlmei • Q. Thai Dinh • \\ Bori Handjiski • Tanja Fischer • Eva M. J. Peters • \\ Burghard F. Klapp • Ralf Paus • Petra C. Arck
}

Received: 7 February 2007 / Revised: 30 April 2007 / Accepted: 29 May 2007 / Published online: 17 July 2007

(C) Springer-Verlag 2007

\begin{abstract}
Emerging research indicates that central-nervous stress perception is translated to peripheral tissues such as the skin not only via classical stress hormones but also via neurotrophins and neuropeptides. This can result in neurogenic inflammation, which is likely to contribute to the triggering and/aggravation of immunodermatoses. Although the existence of such a "brain-skin connection" is supported by steadily increasing experimental evidence, it remains unclear to which extent perceived stress affects the sensory "hardwiring" between skin and its afferent neurons in the corresponding dorsal root ganglia (DRG). In this paper, we provide experimental evidence in a murine model of stress (exposure of C57BL/6 mice to sound stress) that stress exposure, or intracutaneous injection of recombinant nerve growth factor (NGF) to mimic the skin's response to stress, up-regulate the percentage of substance $\mathrm{P}(\mathrm{SP})^{+}$or calcitonin gene-related peptide $(\mathrm{CGRP})^{+}$sensory neurons in
\end{abstract}

R. A. Joachim • Q. T. Dinh • B. Handjiski · T. Fischer

E. M. J. Peters · B. F. Klapp · P. C. Arck

Center of Internal Medicine and Dermatology,

Charité, University Medicine Berlin, Campus Virchow,

Berlin, Germany

A. Kuhlmei

University of Ulm,

Ulm, Germany

R. Paus

Department of Dermatology, University of Schleswig Hostein, University of Luebeck,

Lübeck, Germany

\section{P. C. Arck $(\bowtie)$}

Universitätsmedizin Berlin, Biomedizinisches Forschungszentrum,

R. 2.0549, Augustenburger Platz 1,

13353 Berlin, Germany

e-mail: petra.arck@charite.de

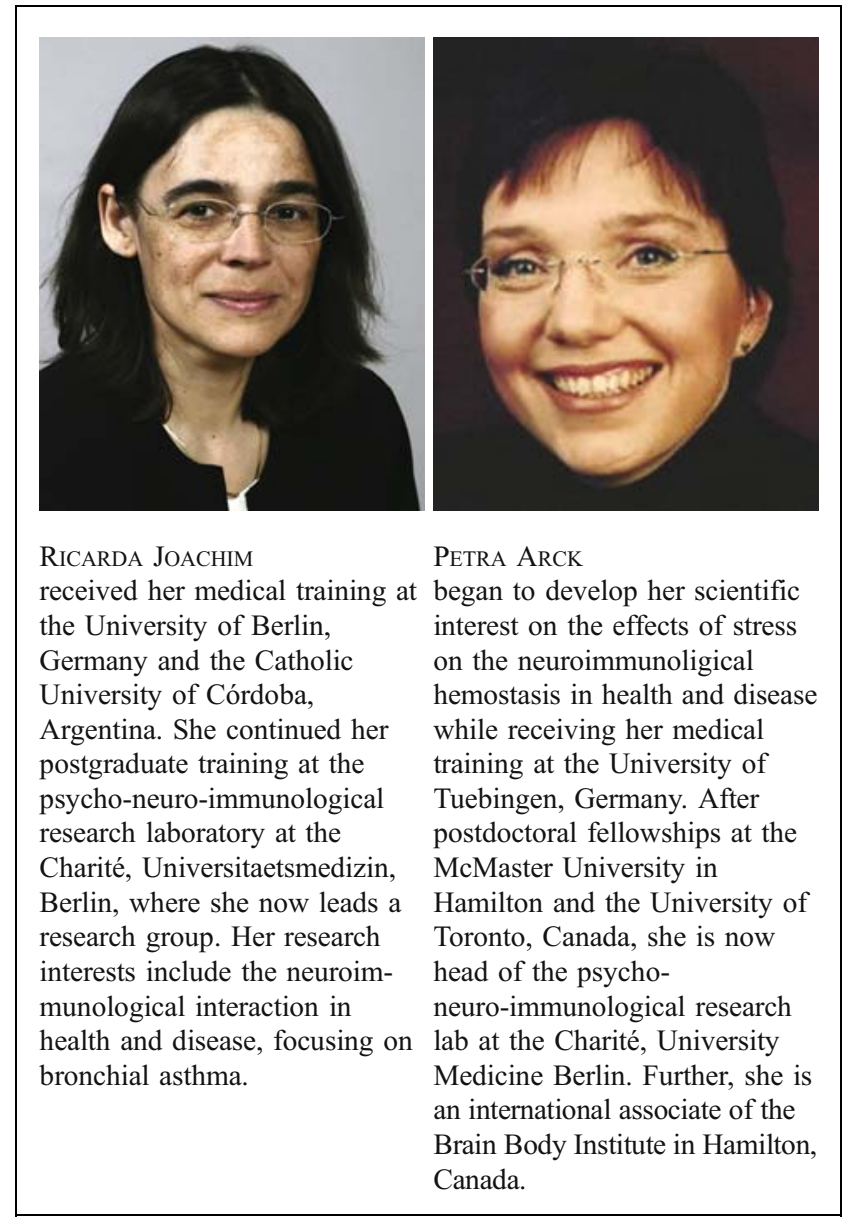

skin-innervating DRG. Further, we show that the number of $\mathrm{SP}^{+}$or $\mathrm{CGRP}^{+}$sensory nerve fibers in the dermis of stressed C57BL/6 mice is significantly increased. Finally, we document that neutralization of NGF activity abrogates 
stress-induced effects on the percentage of $\mathrm{SP}^{+}$and $\mathrm{CGRP}^{+}$ sensory neurons in skin-innervating DRG as well as on dermal sensory nerve fibers. These data suggest that high stress perception results in an intense cross talk between the skin and skin-innervating DRG, which increases the likelihood of NGF-dependent neurogenic skin inflammation by enhancing sensory skin innervation.

Keywords Brain-skin connection · Dorsal root ganglia . Nerve growth factor-dependent pathways · Stress ·

Atopic dermatitis $\cdot$ Hair loss

\section{Introduction}

Emerging research indicates that the central stress perception is translated to the body via stress mediators such as neurotrophins and neuropeptides-besides the traditional notion of a stress-triggered activation of the hypothalamic pituitary adrenal (HPA) axis. In general, such stress-response pathways may be considered as a physiological process of adaptation to the demands of the environment; however, an exceeding stress response has been shown to aggravate or trigger chronic diseases in selected individuals, such as exacerbations of inflammatory bowel disease, atopic diseases, or pregnancy complications [1-5]. High perception of stress influences skin immune homeostasis and may contribute to trigger and/or aggravate immune dermatoses such as atopic dermatitis, psoriasis, alopecia areata (AA), or chronic urticaria [6-8]. Further, stress perception has been suggested as a cause or aggravating factor of telogen effluvium $[9,10]$.

Based on published evidence indicating a link between stress perception and skin diseases [6,7], we previously postulated the existence of the "brain-skin connection" through which the central stress response and peripheral mediators of stress response execution challenge the skin's cell homeostasis and neuro-endocrine-immune equilibrium [11]. The stress-induced increased expression of substance $\mathrm{P}$ (SP) in skin nerve fibers and of cutaneous nerve growth factor (NGF) consequently lead to the development of neurogenic skin inflammation, recognized by its classical features of mast cell degranulation, vasodilatation, and plasma extravasation $[12,13]$. Both SP and NGF are now widely acknowledged as cardinal immunomodulators and prevailing stress markers in different settings [4, 14-16]. NGF, an essential trophic factor for axon sprouting of peptidergic and sympathetic neurons, promotes cross-talk between neuronal cells, glia, and immune cells and facilitates monocyte/macrophage migration through vascular endothelium [17]. During inflammation, NGF is markedly up-regulated in nerves associated with the inflamed area
[18], and NGF levels are increased in inflammatory skin diseases such as psoriasis [19].

Today, established mouse models are on hand to investigate stress effects on skin diseases. Sound stress, which is widely used in neuro-endocrine-immunological research [3, $5,20,21]$, has been demonstrated to induce neurogenic skin inflammation in mice $[4,22]$ and the premature termination of murine hair growth $[2,4]$. Blockade of the neurokinin-1 receptor [2], for which SP has the highest affinity, as well as neutralization of NGF abrogate stress-induced neurogenic inflammation [4]. Interestingly, Aoki and Kawana [23], who employed a mouse model of intermittent foot shock stress, independently supported the existence of a stress-associated brain-skin connection by observing a retardation of hair follicle cycling in stressed mice.

In the skin, sensory nerves derive from the dorsal root ganglion (DRG) and represent the initial somatic portion of the afferent sensory pathway. This cutaneous sensory nervous system comprises a network of fine $\mathrm{C}$-fibers which innervate multiple target structures and play an essential role in inflammation. SP is synthesized in DRG and transported to the terminals of C-fibers located in the dorsal horn of the spinal cord and in the skin [24], where it can be released from sensory nerve fiber endings, e.g., in response to NGF [25]. In-depth analysis of the intricate skin innervation further revealed that-besides $\mathrm{SP}$ - the neuropeptide calcitonin gene-related peptide (CGRP) is localized in primary afferent sensory neurons in bundles as well as single nerve fibers, with distinct distribution patterns and major hair-cycle-associated changes [26, 27]. In the epidermis and around the distal hair follicle and the arrector pili muscle, $\mathrm{CGRP}^{+}$nerve fibers can be detected, whereas $\mathrm{SP}^{+}$nerve fibers are largely restricted to the dermis and subcutis. Compared to telogen skin, the number of CGRP ${ }^{+}$ and $\mathrm{SP}^{+}$single nerve fibers is increased during anagen in skin regions such as the bulge region [27], where the epithelial stem cells of the hair follicle are located. CGRP is a potent vasodilator, mediates weal and flare reaction, and hence contributes to the perpetuation of neurogenic inflammation [28]. Like SP, CGRP can be released in response to peripheral stimuli or by inflammatory mediators like interleukin (IL)-1 or prostaglandins [12]. Peptidergic cutaneous innervation, which is subject to lifelong plasticity, is altered in response to stress, as reflected by an increased number of $\mathrm{SP}^{+}$nerve fibers and an increased expression of NGF [2, 22]. However, to date, no insights are available on cutaneous CGRP expression in response to stress. Further, it remains to be elucidated whether or not NGF-dependent skin inflammation orchestrates neuropeptide expression in DRG and if stress-triggered plasticity of $\mathrm{SP}^{+}$and $\mathrm{CGRP}^{+}$DRG neurons and skin nerve fibers is largely dependent on NGF. 
By employing the technique of retrograde tracing [29], skin-innervating neurons in cervical and thoracic DRG can be identified and allow their phenotypic evaluation. This method, fundamental to map connectivity in the nervous system, may provide insights if and how stress-induced and NGF-dependent skin inflammation orchestrates neuropeptide expression in DRG by altering the relative expression of neuropeptides among traced neurons.

Thus, in the present study, we aimed to investigate

1. whether stress exposure or subcutaneous (s.c.) injection of recombinant NGF (to mimic stress in the absence of sonic stress) affects the percentage of $\mathrm{SP}^{+}$and/or $\mathrm{CGRP}^{+}$sensory neurons in skin-innervating thoracic and cervical DRG in mice.

2. whether an increase in intracutaneous NGF (either as a skin response to stress or as a result of s.c. NGF injection) alters the number of immunohistochemically detectable $\mathrm{SP}^{+}$and/or $\mathrm{CGRP}^{+}$sensory nerve fibers in the dermis.

3. whether neutralization of NGF mitigates or abrogates stress-induced effects on the percentage of $\mathrm{SP}^{+}$ or $\mathrm{CGRP}^{+}$sensory neurons in skin-innervating DRG and/or on $\mathrm{SP}^{+} / \mathrm{CGRP}^{+}$sensory dermal nerve fibers.

\section{Materials and methods}

\section{Animals}

Six- to 8-week-old female C57BL/6 mice were purchased from Charles River (Sulzfeld, Germany) as mice at this age show the most reliable and profound stress response and are in the telogen stage of the hair cycle. The animals were housed in community cages with 12-h light periods and were fed water and mouse chow ad libitum. Animal care and experimental procedures were followed according to guidelines of the respective institutions and conformed to the requirements of the state authority for animal research conduct.

\section{Anagen induction}

Anagen was experimentally induced by depilation, as previously published [30]. Briefly, mice were anesthetized with intramuscular injection of ketamine hydrochloride (Ketanest, Parke-Davis, Freiburg, Germany, $10 \mathrm{mg} / \mathrm{kg}$ body weight) and xylazine hydrochloride (Rompun, Bayer, Leverkusen, Germany, $10 \mathrm{mg} / \mathrm{kg}$ ). Then, a wax/rosin mixture was applied to the dorsal skin of mice with all hair follicles in telogen, as evidenced by the pink back skin color. Peeling off the wax/rosin mixture removes all hair shafts and immediately induces a highly synchronized hair growth.

\section{Retrograde Tracing of DRG}

Mice were anesthetized 9 days post-depilation (p.d.), and a FluoroGold-like tracer (hydroxystilbamidine; Biotium) was applied in five s.c. injections with a total volume of $20 \mu \mathrm{l}$ at a concentration of $1.25 \%\left(100 \mathrm{mg} / 800 \mu \mathrm{l}\right.$ distilled $\left.\mathrm{H}_{2} \mathrm{O}\right)$ in the dorsal skin tissue right below the scapula, covering a total tissue area of about $1 \mathrm{~cm}^{2}$. All animals recovered undisturbed for a postoperative period of 5 days, until day 14 p.d. [4].

\section{Application of stress}

For each experimental setting, a group of C57BL/6 mice $(n=4)$ was exposed to sonic stress for the duration of $24 \mathrm{~h}$ starting on day 14 p.d., when all back skin hair follicles were in late anagen. The time point of stress application in the model of induced anagen was chosen based on earlier experiments, where stress effects on hair cycle and neurogenic inflammation in the skin were examined [2, 4]. In this paper, we aimed to extend the knowledge about stress effects to the level of skin-innervating neurons and influence on neuronal and peripheral CGRP expression. The sonic stress was emitted by a rodent repellent device (Conrad Electronics, Berlin, Germany) at a frequency of 300 Hertz in intervals of $15 \mathrm{~s}$. The stress device was placed into the mouse cage so that the mice could not escape sound perception.

\section{Application of NGF}

A subgroup of nonstressed mice $(n=4)$ was injected subcutaneously on day 14 in the dorsal skin with murine NGF (7S; Roche, Mannheim, Germany) with $10 \mu \mathrm{l} /$ mouse at a concentration of $100 \mu \mathrm{g} / \mathrm{ml}$ phosphate-buffered saline (PBS). The NGF concentration has been selected considering a publication with local NGF application to murine airways [31]. We included this subcutaneous application of NGF to mimic stress-triggered increase in skin NGF. Another subgroup of mice was injected with PBS alone and served as a nonstressed control $(n=4)$.

\section{Application of anti-NGF}

Intraperitoneal application of $200 \mu$ polyclonal rabbit antimouse NGF antibody (Sigma-Aldrich, Munich, Germany) at a dilution of 1:1,500 in PBS was performed in two respective groups of nonstressed control $(n=4)$ or stressed mice $(n=4)$ on days 14 and 15 p.d. According to the 
manufacturer, a 1:4,000 dilution of this antibody blocks bioactivity of $5 \mathrm{ng} / \mathrm{ml} \mathrm{NGF}$.

\section{Tissue preparation}

On day 16 p.d., at the time when control mice are just about to spontaneously enter the anagen/catagen transformation of their depilation-induced hair cycle [32], all mice were killed. The animals were perfusion-fixed retrogradely through the left ventricle with freshly prepared Zamboni's solution ( $2 \%$ paraformaldehyde, $15 \%$ picrinic acid, $0.1 \mathrm{~mol} / 1 \mathrm{PBS}$, pH 7.4) for $5 \mathrm{~min}$. Dissection of the Zamboni-fixed cervical and thoracic DRG (C4 to Th10) was performed by rinses in $0.1 \mathrm{~mol} / \mathrm{l}$ phosphate buffer ( $\mathrm{pH} 7.4$ ) and cryoprotection with $18 \%$ sucrose in $0.1 \mathrm{~mol} / 1$ phosphate buffer overnight. On average, 30 DRGs were harvested per mouse and screened for traced neurons by preparing cryosections of each DRG. Traced DRG could predominantly be identified from C5 to Th3, thus, on average, 14 traced DRG/mouse were available for the preparation of serial sections, and approximately 40 consecutive slides were cut per DRG.

Skin specimens from the neck region of the murine back were harvested parallel to the vertebral line, snap-frozen in liquid nitrogen, and then covered with embedding medium, as described in detail in by Müller-Röver et al. [30]. Cryosections were then processed for immunohistochemistry.

\section{Fluorescence immunohistochemistry of SP and CGRP} in ganglia and skin tissue

Ganglion $(7 \mu \mathrm{m})$ and skin $(14 \mu \mathrm{m})$ sections were air dried for $30 \mathrm{~min}$ and incubated overnight with the corresponding primary antibody at a dilution of 1:400 with a monoclonal rat antimouse SP antibody (Chemicon) and at a dilution of 1:200 with monoclonal rabbit antimouse CGRP antibody (Chemicon). Several washing steps were followed by an incubation of $1 \mathrm{~h}$ at $37^{\circ} \mathrm{C}$ with rhodamineconjugated $\mathrm{F}(\mathrm{ab})_{2}$ fragments of goat antimouse $\mathrm{IgG}$ or goat antirabbit IgG (Jackson ImmunoResearch), respectively, at a Tris-buffered saline (TBS) dilution of 1:200 in $2 \%$ mouse normal serum. All sections were mounted and stored at $-20^{\circ} \mathrm{C}$ until further analysis.

Quantitative histomorphometry and statistical analysis

A fluorescence microscope (Axioplan; Zeiss, Jena, Germany) with appropriate excitation emission filter system for fluorescence induced by rhodamine was used to analyze fluorescence immunohistochemistry (FIH)labeled sections. FluoroGold-positive neurons were identified through a wide-band UV filter. To avoid double counting of neurons, only neurons with an apparent nucleus were counted. Expression of neuropeptides in ganglion sections is indicated as percentage of retrograde traced neurons. Analysis and photo documentation was performed by a digital image analysis system (Spot advanced software, version 3.5.2; Visitron Systems; Puchheim, Germany). Quantification of single dermal nerve fibers was performed by histomorphometry as previously described [33]. From each mouse, two to four different sections were used to analyze at least ten adjacent microscopic fields. This number was raised up to $100 \mathrm{mi}-$ croscopic fields per mouse if there were microscopic fields with no positive nerve-fiber profiles detectable. The data were pooled per mouse and the mean and standard error calculated per group. Nonparametric tests were used because of nonnormal distribution of the results (KolmogorovSmirnov). Significance of differences between groups was determined using the nonparametric Kruskal-Wallis test followed by the Mann-Whitney $U$ test to compare two groups. Differences were judged as significant if the $p$ value was $<0.05$.

\section{Results}

Stress and cutaneous injection of NGF up-regulate the percentage of $\mathrm{SP}^{+}$and $\mathrm{CGRP}^{+}$sensory neurons in skin-innervating DRG

The percentage of $\mathrm{SP}^{+}$and $\mathrm{CGRP}^{+}$sensory neurons in skininnervating DRG was determined among the total number of neurons which could be traced by retrograde labeling. As already shown in pilot experiments in one of our earlier publications [4], the percentage of $\mathrm{SP}^{+}$sensory neurons in skin-innervating DRG was significantly increased in animals exposed to sonic stress compared to nonstressed control animals $(p<0.05$; Fig. 1a; representative photographs are shown in Fig. 2a-d). S.c. injection of recombinant murine NGF in the dorsal skin mimicked the effect of stress on neuronal plasticity in DRG, as the percentage of $\mathrm{SP}^{+}$sensory neurons in skin-innervating DRG was significantly increased in these animals compared to nonstressed control animals $(p<0.05$; Fig. 1a).

Similar results were obtained with respect to $\mathrm{CGRP}^{+}$ sensory neurons in skin-innervating DRG. In the present study, stress exposure resulted in a significantly higher percentage of $\mathrm{CGRP}^{+}$neurons among the skin-innervating neurons localized in spinal DRG compared to nonstressed control mice $(p<0.05)$. Further, s.c. injection of NGF into the dorsal skin also caused an increase in the relative CGRP expression among the traced neurons, akin to stress exposure $(p<0.05$; Fig. 1b, representative photographs are shown in Fig. 2e-h). 
Fig. 1 a Percentage of substance P-positive neurons from total number of retrograde traced neurons in dorsal root ganglia from nonstressed controls, stressed mice, and nonstressed mice subcutaneously injected with NGF. Columns represent mean and SEM per group. b Percentage of CGRPpositive neurons from total number of retrograde traced neurons in dorsal root ganglia from nonstressed controls, stressed mice, and nonstressed mice subcutaneously injected with NGF. Columns represent mean and SEM per group

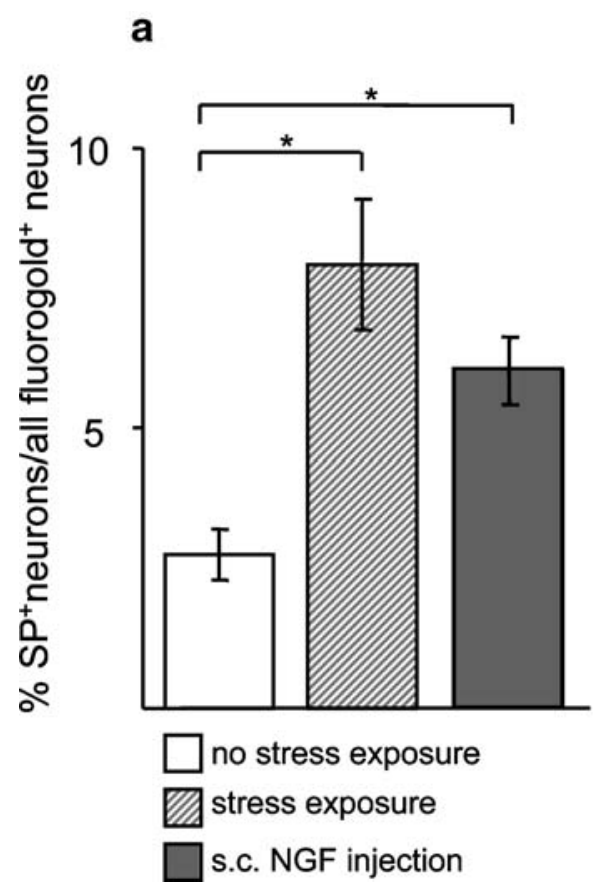

Increased numbers of $\mathrm{SP}^{+}$and $\mathrm{CGRP}^{+}$sensory nerve fibers in the dermis

Besides the effect of stress and/or cutaneous NGF on neuronal plasticity of DRG, we wished to identify the effect of stress exposure or s.c. NGF injection in the dorsal skin on the expression of neuropeptides in the skin. In this paper, we confirmed our pilot data [2] by showing that stress exposure resulted in an increased number of $\mathrm{SP}^{+}$ nerve fibers in the skin, compared to nonstressed mice $(p<$ 0.01 ). In addition, we now provide experimental evidence that local injection of NGF in the dorsal skin led to an increased expression of $\mathrm{SP}^{+}$nerve fibers in the skin, although levels of significance could not be reached when compared to nonstressed control mice (Fig. 3a, representative photographs are shown in Fig. $2 \mathrm{i}$ and j).

Stress exposure resulted in an increased number of $\mathrm{CGRP}^{+}$nerve fibers in the skin compared to nonstressed mice $(p<0.05)$. Further, local injection of recombinant NGF in the dorsal skin led to a significantly increased number of $\mathrm{CGRP}^{+}$nerve fibers in the skin when compared to nonstressed control mice $(p<0.05$; Fig. 3 b; representative photographs are shown in Fig. $2 \mathrm{k}$ and 1 ).

Neutralization of NGF abrogates stress induced effects on the percentage of $\mathrm{SP}^{+}$and $\mathrm{CGRP}^{+}$sensory neurons in skin-innervating DRG

In a second set of experiments, we determined the role of NGF in stress-induced neuronal plasticity of $\mathrm{SP}^{+}$and
$\mathrm{CGRP}^{+}$neurons in retrograde traced neurons of the DRG and nerve fibers in the skin, respectively, by neutralizing NGF in mice, employing an established method of injecting mice with a neutralizing antibody to NGF $[4,34]$. This showed that neutralization of NGF significantly abrogated the effect of stress on the increased percentage of $\mathrm{SP}^{+}$ neurons in DRG $(p<0.05$; Fig. 4 a). Similarly, neutralization of NGF also diminished the stress-triggered increased percentage of $\mathrm{CGRP}^{+}$neurons in DRG $(p<0.05$; Fig. $4 \mathrm{~b})$.

Neutralization of NGF abrogates stress induced effects on numbers of $\mathrm{SP}^{+}$and $\mathrm{CGRP}^{+}$sensory nerve fibers in the dermis

Next, we analyzed the effect of NGF neutralization on $\mathrm{SP}^{+}$ and $\mathrm{CGRP}^{+}$sensory nerve fibers in the dermis and observed that both the stress-triggered increase in $\mathrm{SP}^{+}$ (Fig. 5a) as well as $\mathrm{CGRP}^{+}$nerve fibers in skin (Fig. 5b) were significantly abrogated upon NGF neutralization.

\section{Discussion}

With the present work, we provide experimental evidence that stress exposure and cutaneous NGF modulate the percentage of $\mathrm{SP}^{+}$and $\mathrm{CGRP}^{+}$neurons in skin-innervating DRG. We further show that stress exposure or s.c. injection of NGF up-regulate the number of $\mathrm{CGRP}^{+}$and $\mathrm{SP}^{+}$skin nerve fibers. Lastly, we provide substantiation that the cross talk between neuropeptide expression in skin and DRG is 

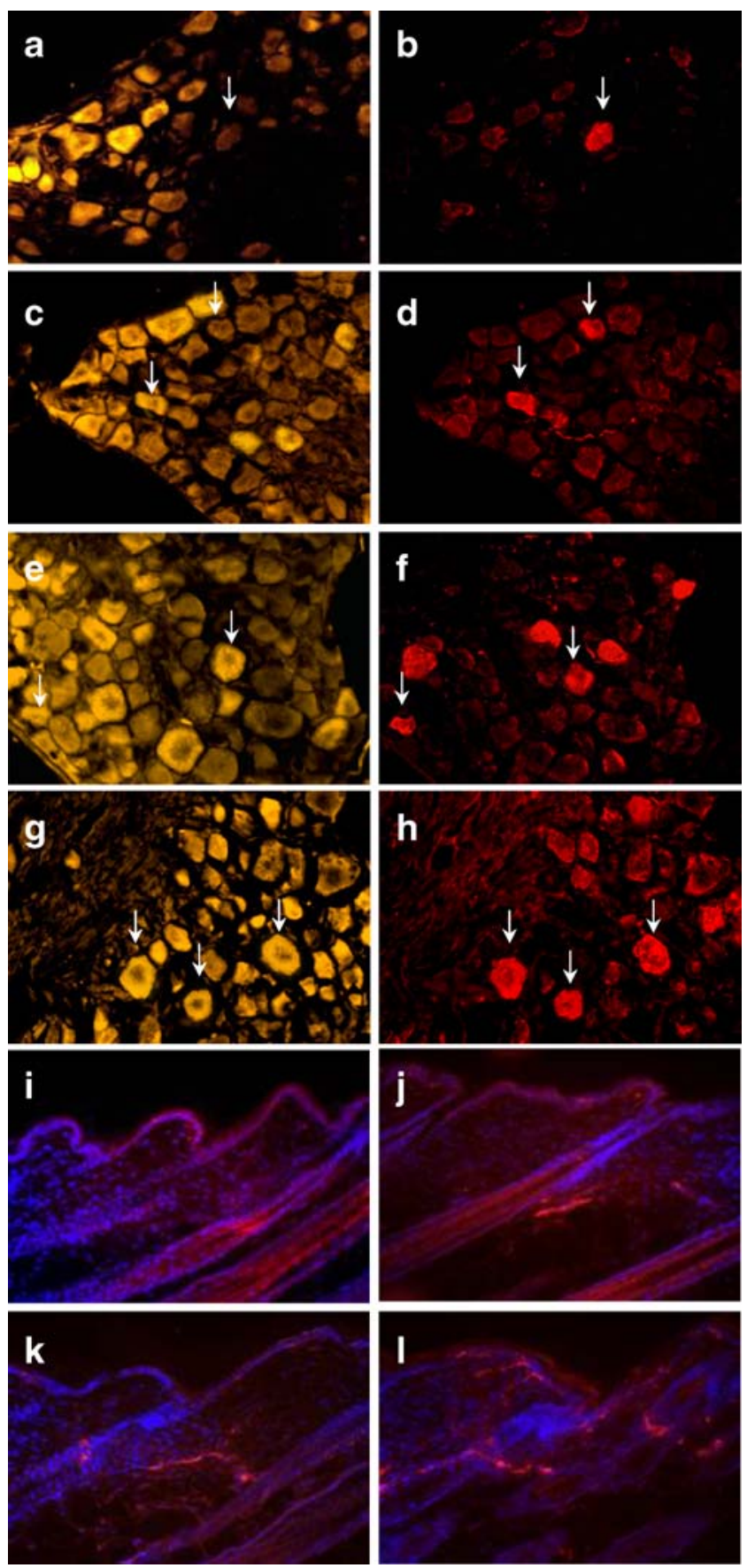

Fig. 2 Representative examples of flourogold-traced dorsal root ganglia from nonstressed (a) and stressed mice (c). b Corresponding area to a using the rhodamine filter to identify substance P (SP)positive neurons. $\mathbf{d}$ Corresponding area to $\mathbf{c}$ using the rhodamine filter to identify SP-positive neurons. Arrows indicate SP-positive neurons. Representative examples of flourogold-traced dorsal root ganglia from nonstressed (e) and stressed mice (g). f Corresponding area to e using the rhodamine filter to identify CGRP-positive neurons. $\mathbf{h}$ Corresponding area to $\mathbf{g}$ using the rhodamine filter to identify CGRP-positive neurons. Arrows indicate CGRP-positive neurons. Immunoreactivity for SP (i, j) and CGRP (k, l). Representative dermis sections from nonstressed controls (i, k) and stressed mice (j, l). Magnification 200× dependent on NGF, as systemic neutralization of NGF prevented stress-triggered alterations of $\mathrm{CGRP}^{+}$and $\mathrm{SP}^{+}$ skin nerve fibers and skin-innervating DRG neurons.

Stress exposure up-regulates the number of CGRP ${ }^{+}$ and $\mathrm{SP}^{+}$skin nerve fibers via NGF-dependent pathways

In the skin, neuropeptides comprise a large family of regulatory molecules including tachykinins, CGRP, vasoactive intestinal peptide (VIP), pituitary adenylate cyclaseactivating polypeptide (PACAP), and others. Their important role in skin health and disease has been investigated intensively (latest reviews [35, 36]). In this context, a wealth of published data is now available on the role of cutaneous SP. Once released into the skin, the tachykinin SP induces inflammation and vasodilatation and activates mast cells [16, 37]. Increased numbers of $\mathrm{SP}^{+}$nerve fibers have been observed in inflammatory skin diseases like atopic dermatitis and psoriasis, as well as in AA and telogen effluvium (recently reviewed in [38]). SP induces adhesion molecule expression (P-selectin, E-selectin, VCAM-1) on skin-isolated human endothelial cells and promotes infiltration with neutrophil and eosinophil granulocytes [39].

Further, emerging research indicates that cutaneous CGRP is released by sensory neurons and modulates vasodilatation, plasma extravasation, as well as several biological functions of epidermal and dermal cells [37]. There is some evidence that immunocompetent cells like macrophages, lymphocytes, neutrophils, mast cells, and Langerhans cells express CGRP receptors indicating the immunomodulating potential for CGRP [40-44]. In the skin, like SP, CGRP exerts vasoactive effects [12]. Clearly, the skin is a crucial barrier protecting the body against external harmful influences [45], which is largely mediated by the skin's immune system, whereas an impaired barrier function is characteristic in immune dermatoses such as atopic dermatitis and psoriasis vulgaris [46, 47]. Thus, an increased release of SP or CGRP in response to stress, as identified in the present study by employing a mouse model, may trigger or aggravate such immune dermatoses and/or AA by challenging skin immune homeostasis and skin barrier function. This notion is supported by studies in humans suggesting that CGRP is involved in the pathophysiogenesis of AA. Hordinsky et al. [48] reported increased neuronal CGRP immunoreactivity around eccrine glands of AA patients, while Meyronet et al. [49] found decreased immunoreactivity of skin and hair follicles in the areas of lesions compared to biopsies from healthy scalp regions. Lower levels of CGRP were detected when comparing biopsies from AA patients with those from healthy controls [50]. Other mediators like the neuropeptide neurotensin [51] or the corticotropin-releasing hormone (CRH) [52] via induction of mast cell degranulation might 
Fig. 3 a Number of substance P-positive nerve fibers per visual field in the dermis from nonstressed controls, stressed mice, and nonstressed mice subcutaneously injected with NGF. Columns represent mean and SEM per group. b Number of CGRP-positive nerve fibers per visual field in the dermis from nonstressed controls, stressed mice, and nonstressed mice subcutaneously injected with NGF. Columns represent mean and SEM per group a

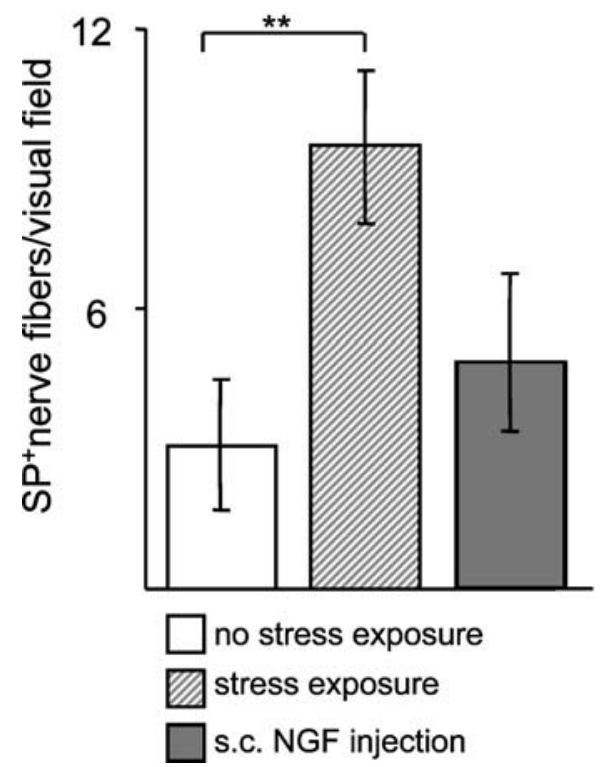

b

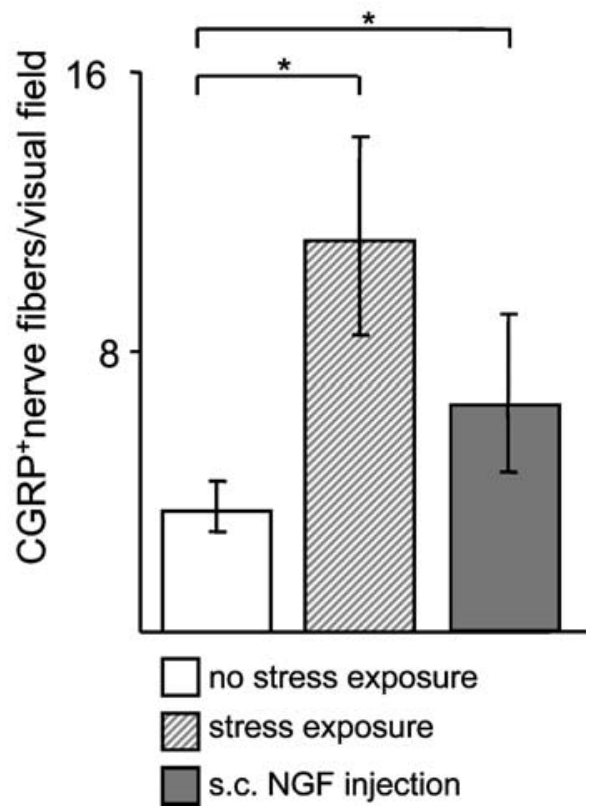

be involved in the pathogenesis of inflammatory skin disorders, especially those exacerbated by stress effects. Future research is needed to identify whether alterations of skin neuropeptide expression in humans may be identified in response to high stress perception.

Stress-triggered cross talk between neuropeptides expression in skin and DRG is dependent on NGF

Neuropeptides are synthesized in the neuronal cell body located in DRG and are transported to the peripheral nerve ending and released under certain conditions like local mechanical or thermical stimulation [53, 54]. As neuropeptides located in cutaneous nerve fibers originate from sensory neurons, we aimed to investigate their neuronal expression. It is well established that increases in the neuropeptides SP and CGRP in the innervating DRG occur after target-tissue damage like inflammation [18] or wounding [55]. In this paper, we were able to show that sonic stress, a stimulus processed and transmitted from the central nervous system, induces neuronal plasticity in skininnervating sensory neurons.

Second aim of the study was to dissect the role of NGF in stress-induced neuropeptide expression in DRG neurons.
Fig. 4 a Percentage of substance P-positive neurons from total number of retrograde traced neurons in dorsal root ganglia from nonstressed controls, stressed mice, and nonstressed mice and the respective groups subcutaneously injected with anti-NGF. Columns represent mean and SEM per group. b Percentage of CGRP-positive neurons from total number of retrograde traced neurons in dorsal root ganglia from nonstressed controls, stressed mice, and nonstressed mice and the respective groups subcutaneously injected with anti-NGF. Columns represent mean and SEM per group
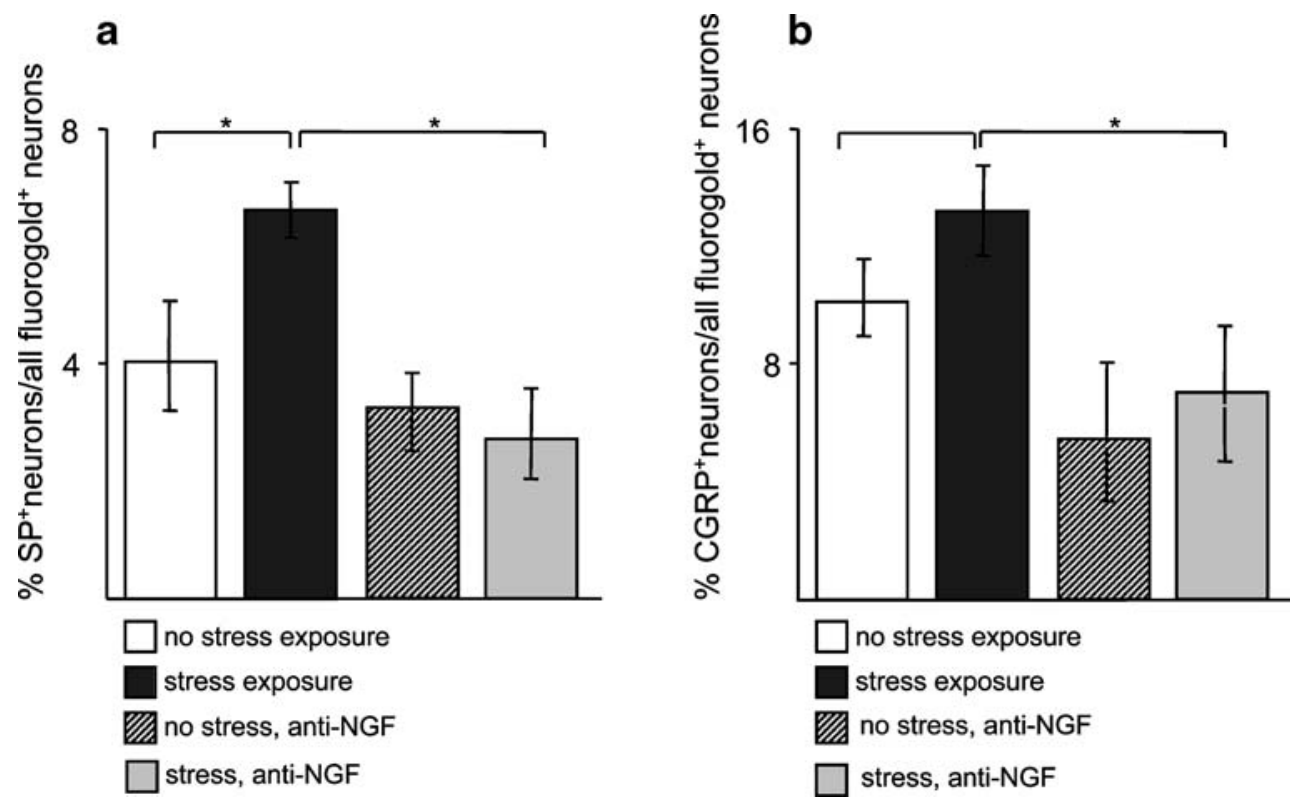
Fig. 5 a Number of substance P-positive nerve fibers per visual field in the dermis from nonstressed controls, stressed mice, and the respective groups subcutaneously injected with antiNGF. Columns represent mean and SEM per group. b Number of CGRP-positive nerve fibers per visual field in the dermis from nonstressed controls, stressed mice, and the respective groups subcutaneously injected with anti-NGF. Columns represent mean and SEM per group a

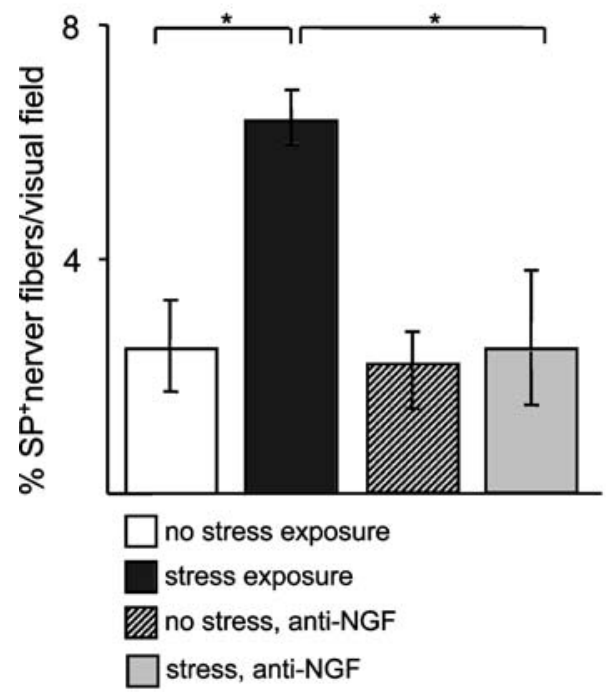

b

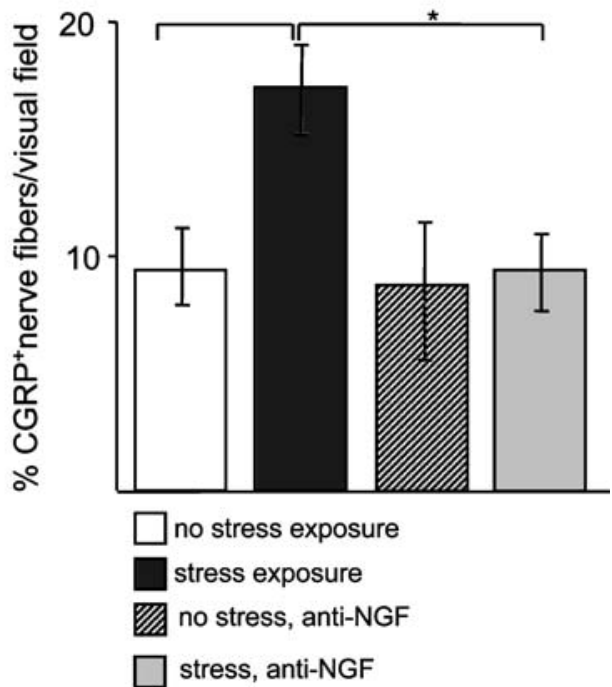

In the recent years, NGF and its ability to induce tachykinin and other neuropeptide expression in DRG have been investigated intensively in animal models in vivo and in vitro. More than 20 years ago, Kessler and Black [25] showed for the first time that neonatal NGF application dramatically increased SP content in DRGs 2 days after application. Similar results were reported by Otten et al. [24] the same year; however, administration of anti-NGF reduced SP content in sensory neurons and nerve fibers in the skin. Local NGF injection into paws or ear skin led to increased preprotachykinin and prepro CGRP mRNA expression in the corresponding DRG and increased SP and CGRP immunoreactivity in corresponding afferent neurons [56]. In the present study, we were able to prove that neuronal plasticity of DRG is under the influence of stress, as the percentages of $\mathrm{CGRP}^{+}$and $\mathrm{SP}^{+}$skininnervating neurons significantly increased, and higher numbers of nerve fibers localized in the skin showed neuropeptide positivity. Such stress-triggered ganglionic and peripheral neuropeptide expression is clearly regulated by NGF, as neutralization of NGF abrogates the stress effects. Further, s.c. application of recombinant NGF mimicked the increase in neuronal neuropeptide expression in DRG. In this paper, no effect of s.c. NGF application could be identified with respect to the number of $\mathrm{SP}^{+}$ cutaneous nerve fibers, which may be attributable to of the experimental approach we chose, such as an impaired bioactivity of NGF by local degradation. However, NGF

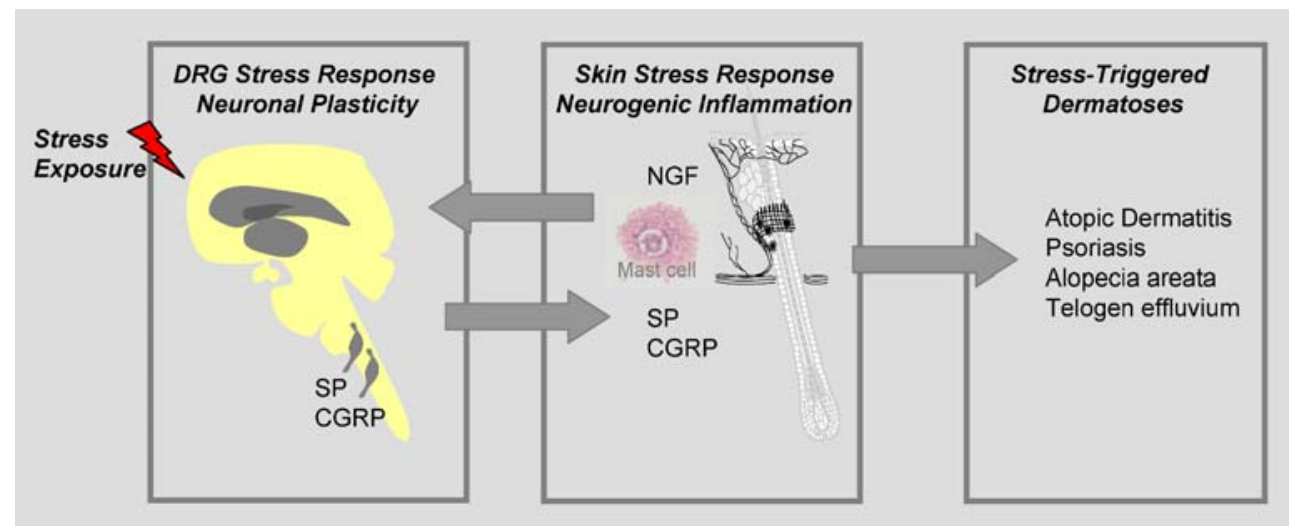

Fig. 6 Hypothetical scenario. After stress or exogenous NGF application, SP and CGRP are up-regulated in skin-innervating neurons located in DRG. This neuronal increase is associated with local neuropeptide increase in skin nerve fibers. Release of these neuropeptides leads to increased neurogenic inflammation, which might not only interfere with hair follicle cycling, subsequently leading to telogen effluvium or an aggravation of alopecia areata, but also deteriorate immune-driven dermatoses such as psoriasis or atopic dermatitis 
application increased the number of $\mathrm{CGRP}^{+}$cutaneous nerve fibers. This result is in line with findings from Albers et al. [57] who found increased sensory innervation in the skin of NGF-overexpressing mice.

Together with the finding that anti-NGF application abrogated stress-induced effects on neuropeptide expression in retrograde traced neurons and local nerve fibers, our data strongly suggest a stress-mediating role for NGF. Probably, stress through the demonstrated neuropeptide release and neurogenic inflammation might impair the skin barrier function by increasing vascular permeability and/or influencing local immunocompetent cell populations like dendritic cells. This question has to be investigated in the future.

Finally, a hypothetical scenario based on our recent findings is drawn in Fig. 6. After stress or exogenous NGF application, SP and CGRP are up-regulated in skininnervating neurons located in DRG. This neuronal increase is associated with local neuropeptide increase in skin nerve fibers. Release of these neuropeptides leads to increased neurogenic inflammation, which might not only interfere with hair follicle cycling, subsequently leading to telogen effluvium or an aggravation of AA, but also deteriorate immune-driven dermatoses such as psoriasis or atopic dermatitis.

Acknowledgments This work was supported by grants from the German Research Foundation (Deutsche Forschungsgemeinschaft; Ar 232/14-2; Pa 345/11-2) and the Charite (UFF 99-648). We thank R. Pliet, E. Hagen, P. Moschansky, and P. Busse for their excellent technical assistance.

\section{References}

1. Levenstein S, Prantera C, Varvo V, Scribano ML, Berto E, Andreoli A, Luzi C (1994) Psychological stress and disease activity in ulcerative colitis: a multidimensional cross-sectional study. Am J Gastroenterol 89:1219-1225

2. Arck PC, Handjiski B, Peters EMJ, Peter AS, Hagen E, Fischer A, Klapp BF, Paus R (2003) Stress inhibits hair growth in mice by induction of premature catagen development and deleterious perifollicular inflammatory events via neuropeptide substance Pdependent pathways. Am J Pathol 162:803-814

3. Joachim RA, Quarcoo D, Arck PC, Herz U, Renz H, Klapp BF (2003) Stress enhances airway reactivity and airway inflammation in an animal model of allergic bronchial asthma. Psychosom Med 65:811-815

4. Peters EMJ, Handjiski B, Kuhlmei A, Hagen E, Bielas H, Braun A, Klapp BF, Paus R, Arck PC (2004) Neurogenic inflammation in stress-induced termination of murine hair growth is promoted by nerve growth factor. Am J Pathol 165:259-271

5. Blois SM, Joachim R, Kandil J, Margni R, Tometten M, Klapp BF, Arck PC (2004) Depletion of CD8+ cells abolishes the pregnancy protective effect of progesterone substitution with dydrogesterone in mice by altering the $\mathrm{Th} 1 / \mathrm{Th} 2$ cytokine profile. J Immunol 172:5893-5899
6. Wright RJ, Cohen RT, Cohen S (2005) The impact of stress on the development and expression of atopy. Curr Opin Allergy Clin Immunol 5:23-29

7. Al'Abadie MS, Kent GG, Gawkrodger DJ (1994) The relationship between stress and the onset and exacerbation of psoriasis and other skin conditions. Br J Dermatol 130:199-203

8. Katsarou-Katsari A, Singh LK, Theoharides TC (2001) Alopecia areata and affected skin CRH receptor upregulation induced by acute emotional stress. Dermatology 203:157-161

9. Panconesi E, Hautmann G (1996) Psychophysiology of stress in dermatology. The psychobiologic pattern of psychosomatics. Dermatol Clin 14:399-421

10. Botchkarev VA (2003) Stress and the Hair Follicle: Exploring the Connections. Am J Pathol 162:709-712

11. Paus R, Theoharides TC, Arck PC (2006) Neuroimmunoendocrine circuitry of the 'brain-skin connection'. Trends Immunol 27:32-39

12. Holzer P (1998) Neurogenic vasodilatation and plasma leakage in the skin. Gen Pharmacol 30:5-11

13. Kiernan JA (1972) The involvement of mast cells in vasodilatation due to axon reflexes in injured skin. Q J Exp Physiol Cogn Med Sci 57:311-317

14. Aloe L, Bracci Laudiero L, Alleva E, Lambiase A, Micera A, Tirassa P (1994) Emotional stress induced by parachute jumping enhances blood nerve growth factor levels and the distribution of nerve growth factor receptors in lymphocytes. Proc Natl Acad Sci U S A 91:10440-10444

15. Tometten M, Klapp BF, Joachim R, Fest S, Zenclussen AC, Peters EM, Hertwig K, Arck PC (2004) Nerve growth factor and its functional receptor TrkA are up-regulated in murine decidual tissue of stress-triggered and substance P-mediated abortion. Am J Reprod Immunol 51:86-93, DOI 10.1046/j.8755-8920.2003. 00123

16. Singh LK, Pang X, Alexacos N, Letourneau R, Theoharides TC (1999) Acute immobilization stress triggers skin mast cell degranulation via corticotropin releasing hormone, neurotensin, and substance P: A link to neurogenic skin disorders. Brain Behav Immun 13:225-239

17. Levi-Montalcini R, Skaper SD, Dal Toso R, Petrelli L, Leon A (1996) Nerve growth factor: from neurotrophin to neurokine. Trends Neurosci 19:514-520

18. Donnerer J, Schuligoi R, Stein C (1992) Increased content and transport of substance $\mathrm{P}$ and calcitonin gene-related peptide in sensory nerves innervating inflamed tissue: evidence for a regulatory function of nerve growth factor in vivo. Neuroscience 49:693-698

19. Bracci-Laudiero L, Pincelli C (1995) Nerve growth factor is increased in psoriatic skin. J Invest Dermatol 105:854-855

20. Qiu BS, Vallance BA, Blennerhassett PA, Collins SM (1999) The role of CD4+ lymphocytes in the susceptibility of mice to stress-induced reactivation of experimental colitis. Nat Med 5: $1178-1182$

21. Pincus-Knackstedt MK, Joachim RA, Blois SM, Douglas AJ, Orsal AS, Klapp BF, Wahn U, Hamelmann E, Arck PC (2006) Prenatal stress enhances susceptibility of murine adult offspring toward airway inflammation. J Immunol 177:8484-8492

22. Peters EMJ, Kuhlmei A, Tobin DJ, Muller-Rover S, Klapp BF, Arck PC (2005) Stress exposure modulates peptidergic innervation and degranulates mast cells in murine skin. Brain Behav Immun 19:252-262

23. Aoki E ST, Kawana S. (2003) Intermittent foot shock stress prolongs the telogen stage in the hair cycle of mice. Exp Dermatol 12:371-377

24. Otten U, Goedert M, Mayer N, Lembeck F (1980) Requirement of nerve growth factor for development of substance P-containing sensory neurones. Nature 287:158-159 
25. Kessler JA, Black IB (1980) Nerve growth factor stimulates the development of substance $\mathrm{P}$ in sensory ganglia. Proc Natl Acad Sci U S A 77:649-652

26. Botchkarev VA, Eichmuller S, Peters EM, Pietsch P, Johansson O, Maurer M, Paus R (1997) A simple immunofluorescence technique for simultaneous visualization of mast cells and nerve fibers reveals selectivity and hair cycle-dependent changes in mast cell-nerve fiber contacts in murine skin. Arch Dermatol Res 289:292-302

27. Peters EMJ, Botchkarev VA, Botchkareva NV, Tobin DJ, Paus R (2001) Hair-cycle-associated remodeling of the peptidergic innervation of murine skin, and hair growth modulation by neuropeptides. J Invest Dermatol 116:236-245

28. Wallengren J, Hakanson R (1987) Effects of substance P, neurokinin $\mathrm{A}$ and calcitonin gene-related peptide in human skin and their involvement in sensory nerve-mediated responses. Eur $\mathrm{J}$ Pharmacol 143:267-273

29. Dinh QT, Groneberg DA, Mingomataj E, Peiser C, Heppt W, Dinh S, Arck PC, Klapp BF, Fischer A (2003) Expression of substance P and vanilloid receptor (VR1) in trigeminal sensory neurons projecting to the mouse nasal mucosa. Neuropeptides 37:245-250

30. Muller-Rover S, Handjiski B, van der Veen C, Eichmuller S, Foitzik K, McKay IA, Stenn KS, Paus R (2001) A comprehensive guide for the accurate classification of murine hair follicles in distinct hair cycle stages. J Invest Dermatol 117:3-15

31. Braun A, Quarcoo D, Schulte-Herbrüggen O, Lommatzsch M, Hoyle G, Renz H (2001) Nerve Growth Factor Induces Airway Hyperresponsiveness in Mice. Int Arch Allergy Immunol 124:205-207

32. Stenn KS, Paus R (2001) Controls of hair follicle cycling. Physiol Rev 81:449-494

33. Botchkarev VA, Eichmüller S, Johansson O, Paus R (1997) Hair cycle-dependent plasticity of skin and hair follicle innervation in normal murine skin. J Comp Neurol 386:379-395

34. Tometten M, Blois S, Kuhlmei A, Stretz A, Klapp BF, Arck PC (2006) Nerve growth factor translates stress response and subsequent murine abortion via adhesion molecule-dependent pathways. Biol Reprod 74:674-683

35. Roosterman D, Goerge T, Schneider SW, Bunnett NW, Steinhoff M (2006) Neuronal control of skin function: the skin as a neuroimmunoendocrine organ. Physiol Rev 86:1309-1379

36. Hendrix S, Peters E (2007) Neuronal plasticity and neuroregeneration in the skin-The role of inflammation. J Neuroimmunol 184:113-126

37. Scholzen TE, Steinhoff M, Bonaccorsi P, Klein R, Amadesi S, Geppetti P, Lu B, Gerard NP, Olerud JE, Luger TA, Bunnett NW, Grady EF, Armstrong CA, Ansel JC (2001) Neutral endopeptidase terminates substance P-induced inflammation in allergic contact dermatitis. J Immunol 166:1285-1291

38. Steinhoff M, Stander S, Seeliger S, Ansel JC, Schmelz M, Luger T (2003) Modern aspects of cutaneous neurogenic inflammation. Arch Dermatol 139:1479-1488

39. Smith C, Barker J, Morris R, MacDonald D, Lee T (1993) Neuropeptides induce rapid expression of endothelial cell adhesion molecules and elicit granulocytic infiltration in human skin. J Immunol 151:3274-3282

40. Wang F, Millet I, Bottomly K, Vignery A (1992) Calcitonin generelated peptide inhibits interleukin 2 production by murine $\mathrm{T}$ lymphocytes. J Biol Chem 267:21052-21057
41. McGillis J, Humphreys S, Reid S (1991) Characterization of functional calcitonin gene-related peptide receptors on rat lymphocytes. J Immunol 147:3482-3489

42. Vignery A, Wang F, Ganz MB (1991) Macrophages express functional receptors for calcitonin-gene-related peptide. J Cell Physiol 149:301-306

43. Kitabatake Y, Kawamura S, Yamashita M, Okuyama K, Takayanagi M, Ohno I (2004) The expression of mRNA for calcitonin gene-related peptide receptors in a mucosal type mast cell line, RBL-2H3. Biol Pharm Bull 27:896-898

44. Hosoi J, Murphy GF, Egan CL, Lerner EA, Grabbe S, Asahina A, Granstein RD (1993) Regulation of Langerhans cell function by nerves containing calcitonin gene-related peptide. Nature 363:159-163

45. Elias PM, Wood LC, Feingold KR (1999) Epidermal pathogenesis of inflammatory dermatoses. Am J Contact Dermat 10: $119-126$

46. Chamlin SL, Kao J, Frieden IJ, Sheu MY, Fowler AJ, Fluhr JW, Williams ML, Elias PM (2002) Ceramide-dominant barrier repair lipids alleviate childhood atopic dermatitis: Changes in barrier function provide a sensitive indicator of disease activity. J Am Acad Dermatol 47:198-208

47. Ghadially R, Reed JT, Elias PM (1996) Stratum corneum structure and function correlates with phenotype in psoriasis. J Investig Dermatol 107:558-564

48. Hordinsky M, Kennedy W, Wendelschafer-Crabb G, Lewis S (1995) Structure and function of cutaneous nerves in alopecia areata. J Invest Dermatol 104:28S-29S

49. Meyronet D, Jaber K, Gentil-Perret A, Cambazard F, Misery L (2003) Decreased CGRP staining in alopecia areata. Brit J Dermatol 149:422-424

50. Rossi R, Del Bianco E, Isolani D, Baccari MC, Cappugi P (1997) Possible involvement of neuropeptidergic sensory nerves in alopecia areata. Neuroreport 8:1135-8

51. Donelan J, Boucher W, Papadopoulou N, Lytinas M, Papaliodis D, Dobner P, Theoharides TC (2006) Corticotropin-releasing hormone induces skin vascular permeability through a neurotensin-dependent process. Proc Natl Acad Sci 103:7759-7764

52. Theoharides TC, Singh LK, Boucher W, Pang X, Letourneau R, Webster E, Chrousos G (1998) Corticotropin-releasing hormone induces skin mast cell degranulation and increased vascular permeability, a possible explanation for its proinflammatory effects. Endocrinology 139:403-413

53. Lu C-L, Pasricha PJ, Hsieh J-C, Lu R-H, Lai C-R, Wu L-L, Chang F-Y, Lee S-D (2005) Changes of the neuropeptides content and gene expression in spinal cord and dorsal root ganglion after noxious colorectal distension. Regul Pept 131:66-73

54. Siney L, Brain SD (1996) Involvement of sensory neuropeptides in the development of plasma extravasation in rat dorsal skin following thermal injury. Br J Pharmacol 117:1065-1070

55. Cruise BA, Xu P, Hall AK (2004) Wounds increase activin in skin and a vasoactive neuropeptide in sensory ganglia. Dev Biol 271:1-10

56. Amann R, Sirinathsinghji DJS, Donnerer J, Liebmann I, Schuligoi R (1996) Stimulation by nerve growth factor of neuropeptide synthesis in the adult rat in vivo: bilateral response to unilateral intraplantar injections. Neurosci Lett 203:171-174

57. Albers K, Wright D, Davis B (1994) Overexpression of nerve growth factor in epidermis of transgenic mice causes hypertrophy of the peripheral nervous system. J Neurosci 14:1422-1432 